\title{
MULTIPLE PREGNANCY, ANTENATAL CARE PROBLEMS AND THEIR SOLUTIONS RESULTS
}

\author{
Iryna Nikitina \\ Department of Obstetrics and Gynaecology \\ Sumy State University \\ 2 Rimsky-Korsakov str., Sumy, Ukraine, 40007 \\ nikitina-med@mail.ru \\ Natalya Kalashnyk \\ Department of Obstetrics and Gynaecology \\ Sumy State University \\ 2 Rimsky-Korsakov str., Sumy, Ukraine, 40007 \\ vladka24091977@mail.ru \\ Svitlana Smiian \\ Department of Obstetrics and Gynaecology \\ Sumy State University \\ 2 Rimsky-Korsakov str., Sumy, Ukraine, 40007 \\ smiyani@ukr.net \\ Tatyana Babar \\ Department of Obstetrics and Gynaecology \\ Sumy State University \\ 2 Rimsky-Korsakov str., Sumy, Ukraine, 40007 \\ gin@med.sumdu.edu.ua \\ Maryna Bolotna \\ Department of Obstetrics and Gynaecology \\ Sumy State University \\ 2 Rimsky-Korsakov str., Sumy, Ukraine, 40007 \\ gin@med.sumdu.edu.ua \\ Alina Popova \\ Department of Obstetrics and Gynaecology \\ Sumy State University \\ 2 Rimsky-Korsakov str., Sumy, Ukraine, 40007 \\ gin@med.sumdu.edu.ua
}

\footnotetext{
Abstract

Aim of the work. The aim of our study is to summarize the course of pregnancy and delivery in multiple pregnancies in modern conditions.

Materials and methods. The analysis of the pregnancy course, labor and perinatal outcomes was carried out in 182 women with diochorionic and 86 patients with monochorionic twins and 40 women with a singleton pregnancy. Multiple pregnancies were proved to be a monochorionic $(\mathrm{MH})$ and diochorionic $(\mathrm{DH})$ that is a high risk factor for perinatal complications. The research included clinical and laboratory examination metods, study of hormonal function of fetoplacental complex (FPC), transvaginal ultrasound examination. Statistical processing of data was carried out using the package of applied programs Microsoft Office Excel 2010 and StatSoft Statistica 6. 1.

Results and discussion. In the analysis of pregnancy the abortion threat was detected in 51 (59.3\%) women with monochorionic and 34 (62\%) women with diochorionic twins. Preeclampsia during pregnancy complicated in $26(30.2 \%)$ and 43 (23.6\%) patients, with monochorionic and diochorionic placentation type, respectively. A frequent complication of both the $\mathrm{MH}$ and $\mathrm{DH}$ twins was anemia, which was found in more than half of the surveyed (56.9 and $51 \%$, respectively). Delivery in the case of multiple pregnancies is also much more complicated and is terminated surgically three times more often than in singleton births. The most fre-
} 
quent indications for operative delivery were first breech fetus, fetal distress during pregnancy and childbirth, FGR, severe preeclampsia, premature detachment of normally situated placenta, anomalies of labor activity. These perinatal losses were equally observed both after cesarean section and after spontaneous labor and amounted to $4 \%$ at monochorionic and $1.7 \%$ at diochorionic twins.

The frequency dependence of identified lesions at pregnancy with twins was established by chorionic. Careful observation of the patients with multiple pregnancies in the antenatal period, the prevention of the most frequent complications, ultrasound monitoring of early pregnancy allows a differentiated approach to the management of pregnancy and childbirth, helping to reduce perinatal morbidity and mortality.

Conclusions. Multiple pregnancies occur with a large number of complications compared with singletons. Thus, the increase of frequency of multiple pregnancy influences the obstetric and perinatal indicators, pushing this issue in a number of the most pressing issues of modern obstetrics and perinatology.

Keywords: multiple pregnancies, miscarriage, threat of premature birth, transvaginal ultrasonography.

\section{Introduction}

Over the past two decades, the world has seen an increase in the frequency of multiple pregnancies (MP), which is still considered to be a specific biological constant $[1,2]$. Statistical research in developed countries has shown that the multiple pregnancy rates continue to increase. Now the twins births occur at $1.4-2.4 \%$ and iatrogenic multiple pregnancy is $30-80 \%$ of multiple pregnancies. In $60-70 \%$ of cases this is due to the frequent use of ovulation induction, superovulation in assisted reproductive technologies (ART), including vitro fertilization (IVF) and embryo transferring (ET) $[4,9]$. When discussing the safety of these technologies special attention is paid to the problem of the health quality of children born after IVF. With the developing reproductive technologies that increase the number of BS, the number of factors that may adversely affect the course of pregnancy, maternal morbidity (increase in 3-7 times) and material costs associated with it also increases. The last are increasing by more than $40 \%$ if compared with a singleton pregnancy [1, 4]. During fetal development a set of factors influence the embryo and fetus that are dangerous for normal development. Firstly, barren women come very late in the reproduction process (more than half of the surveyed women were aged over 30 years), in connection with which the risk of children's birth with congenital malformations dramatically increases. Secondly, a significant number of subjects $(60.5 \%)$ had severe somatic pathology which required drug therapy. In addition, all women received hormone therapy to stimulate superovulation and (or) the continuation of the pregnancy that also had some adverse effects on fetal development $[7,9,12]$.

Multiple pregnancies have a high rate of anti-perinatal maternal complications, fetal and neonatal deaths of children, perinatal morbidity. This perinatal loss and the incidence of multiple pregnancies increase in proportion to the number of fetuses developing [5,6]. Pathogenesis of perinatal pathology in multiple gestations cannot be fully explored. And so far there are no clear data about the features of the formation and the functional state of feto-placental complex, depending on the cause and nature of the twins, the methods for assessing the embryo's state, the monitoring of fetal development, prenatal care and prognosis of perinatal outcomes at pregnant women with a multiple pregnancy are insufficiently developed.

Multiple pregnancies are accompanied by a large number of complications in pregnancy and childbirth: preeclampsia, miscarriage, premature labor, anemia, gestational diabetes, fetal growth retardation, feta-fetal transfusion syndrome, abnormal position of the fetus and placenta location, poly hydramnios and oligo hydramnios, bleeding that leads to maternal morbidity and mortality. It was found that the development of preeclampsia in induced multiple pregnancies occurs twice as often as in the population depending on the type of placentation. At trichorionic triamniotichny triplet's preeclampsia was observed in $80 \%$, at duo amniotic monochorionic twins - in $66.6 \%$, at duo amniotic diochorionic twins - in $28.1 \%$ of cases. Patients with BV are in high risk of perinatal complications [8-11]. Perinatal morbidity and mortality in twins depends on chorionic. According to the studies, the level of perinatal mortality is much higher in monochorionic twins than in diochorionic ( $5 \%$ and $2 \%$, respectively), fetus abortion at birth, incidence of preterm birth before 32 weeks of pregnancy with monochorionic is $10 \%$ compared to $5 \%$ at diochorionic twins. The 
frequency of spontaneous abortion in the period from 11 to 24 week with diochorionic twins is $2 \%$, with monochorionic - about $10 \%$ [7].

The questions of early diagnosis of multiple pregnancies, eliminating the obstetric complications, the increase in the duration of pregnancy, in order to avoid complications in childbirth and optimal care for newborns, are the main tasks in the management of multiple pregnancies and the key to solving the problem of reducing perinatal losses during multiple pregnancies. A separate problem comprises the conducting of multiple births, where the question of choosing the tactics and timing the delivery is closely linked to the organizing the obstetric care, material equipment and staffing the medical institutions that can take on the responsibility for the delivery of pregnant women with multiple pregnancies $[1,13]$.

\section{Aim}

The aim of our study: to summarize the course of pregnancy and delivery in multiple pregnancies in modern conditions.

\section{Materials and methods}

Research was conducted at the Sumy Regional Clinical Perinatal Center during 2014-2016 years. In the process of scientific research we examined 182 pregnant women with diochorionic twins, composition I group, and 86 patients with monochorionic (85 duo amniotic and one mono amniotic), they were included in group II, and controlled group consisted of 40 women with a singleton pregnancy. A specially designed card was filled in for each of the examinees. The bulk of the studied pregnant women with multiple pregnancies were women aged 26 to 30 years (52.8\%). At the age of 30 and over there were about 65 patients of group I (35.7\%) and 30 women of group II $(34.8 \%)$. Percentage of first pregnant and multiparous in both groups was identical and comprised respectively: group I - $63 \%$ nulliparous, multiparous - $37 \%$; group II $68 \%$ - and $32 \%$ of nulliparous - multiparous. In $65(35.7 \%)$ patients with adiochorionic twins pregnancy was induced, among them in 52 (28.6\%) women pregnancy came after IVF [12, 16]. In addition to clinical examination and conventional laboratory tests, monitoring of early pregnancy with ultrasound was carried out. Ultrasonography (US) in a period of 10-13 weeks was carried out to determine certain viability, number of fruits, chorionic, nuchal translucency, congenital malformations, and the exact gestational age. In the later period of pregnancy (18-22 weeks) was carried ultrasound photometry, placenta studying included measurements of its thickness and the assessment of the degree of maturity according to Prof. Grannum and other authors (1979) [14, 15]. In addition, during ultrasound was determined the amount of amniotic fluid, the thickness of the membrane between embryo, the placement of attached umbilical cord, as well as the sex of the fetus. All women within the period of 26 to 34 weeks of pregnancy and abortion with presenting symptoms underwent examination by ultrasonic cervimeter. To determine the status of embryo and placental and fetal hemodynamics was used doppler blood flow in the umbilical artery and embryo's middle cerebral artery [6, 9]. Ultrasound examinations were performed with a modern ultrasound scanner "SONOACE X8" (Medison, Korea) using transvaginal transducer $6.5 \mathrm{MHz}$, convex sensors 3,5 and $5 \mathrm{MHz}$ in a two echo regime using color Doppler analyzer [17, 18, 19]. Newborn condition at birth was estimated according to Apgar and Silverman, evaluated the severity of newborns' malnutrition. In the neonatal period was carried examination by neuron sonographer (NSG), track psychomotor development was studied within the first six months of life in terms of infants.

Statistical processing of data was carried out using the package of applied programs Microsoft Office Excel 2010 and StatSoft Statistica 6.1 [3].

\section{Results of research}

Ultrasound examination in I trimester of pregnancy sets the type of placentation at pregnant women with multiple pregnancy. The differential diagnosis of mono- and diochorionic twins in early pregnancy is based on determining the amount of fetal eggs, embryos in the egg, as well as on the identification of T or Y-signs during careful studying the partition between fetuses (Fig. 1, 2). In one case the lack of the partition between fetuses was the basis for the mono amniotic diagnosis 
of monochorionic twins; this pregnant woman was included in the group II of examined patients. Thickness of the partition between fetuses, which is one of the diagnostic criteria of chorionic vibrates from 0.8 to $1.1 \mathrm{~mm}$ with monochorionic twins and from 1.4 to $2.8 \mathrm{~mm}$ with diochorionic placentation type. In the later stages of pregnancy, additional criteria such as gender placentation of fetus, number of placentas, as well as differences in their maturation appear.

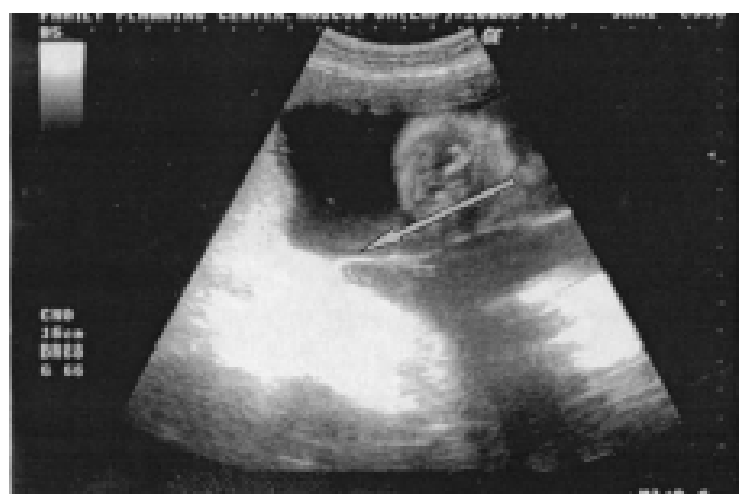

Fig. 1. T-sign with monochorionic twins

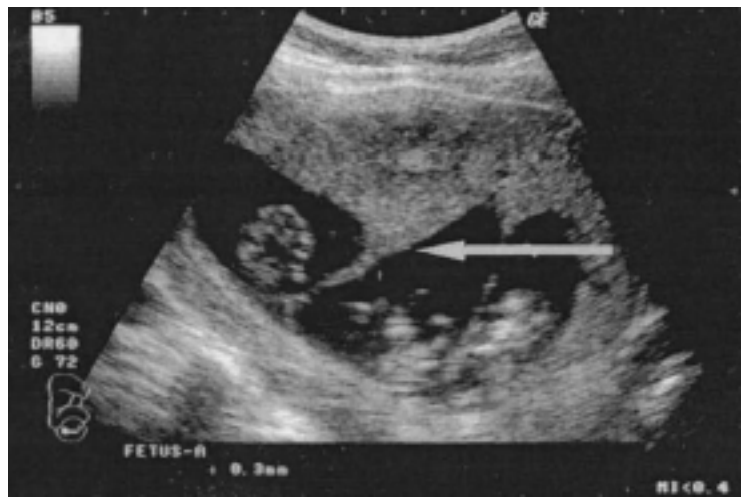

Fig. 2. $\lambda$-sign with monohorionic twins

During ultrasound examination of the pregnant patients of Group II meningeal attachment cord was diagnosed in 16 fetuses with monochorionic twins, including 12 cases and in other two in both fetuses. All 16 fetuses is combined by fetal growth retardation (FGR): I degree - in one, II of degree - in 7 and III - 8 fetuses apparently due to the formation of abnormal placental circulation at a pathology of the umbilical cord attachment. In diochorionic pregnancy the rate of complications was $8.6 \%$. Polyhydramnios was found in $26.2 \%$ of fetuses in group II, including during trimester II - 18 (40 \%) and III - 27 (60\%). Note that expressed polyhydramnios (amniotic vertical column of 120 to $155 \mathrm{~mm}$ ) was found in six pregnant. The same percentage of observations diagnosed hypamnion $-41(23.8 \%$ ) fetus, which often was found out in the III trimester (75.6 against $24.4 \%$ in the II trimester).

Complex pathologic ultrasonic parameters (dissociation expressed in fetal weight - from 15 to $55 \%$, polyhydramnios/hypamnion) allowed the diagnosis of prenatally syndrome feto-fetal transfusion (SFFT) in 20 patients of group II within 18-30 weeks. An important predictor of the development of SFFT, in our opinion, is the difference in the thickness of the placenta in its various departments, which are already diagnosed with ultrasound at 15-20 week of pregnancy, and was a sign of edema of the placenta, which belonged to the recipient.

In the future, with the growth of polyhydramnios in the recipient and hypamnion in the fetus, the donor will have some reduction in the thickness of this part of the placenta due to its mechanical compression of the large volume of amniotic fluid. Severe SFFT was found in eight 
patients of group II in the II trimester $-9.3 \%$ of pregnant women, which led to late miscarriage in five cases (including one patient with mono amniotic twins), antenatal death of both fetuses in one pregnant and one twin in two patients within the period of 28-30 weeks. Pathognomonic echo feature of heavy SFFT was the presence of a large bladder of the recipient's fetus on the background of expressed polyhydramnios and no visualization of the bladder in the donor's fetus, which was characterized by decreased motor activity on the background of marked hypamnion. Severe SFFT was characterized by abnormal blood flow in the umbilical artery in both fetuses when systolic-diastolic ratio (SDR) was bigger in 3.3, and was more expressed in the recipient's fetus that has the reversed blood flow. In the donor's fetus it is caused by placental pathology development, the recipient's fetus blood disorders are associated with compression of the umbilical cord due to polyhydramnios.

\section{Discussion}

In the analysis of pregnancy the abortion threat was detected in 51 (59.3\%) women with monochorionic and $34(62 \%)$ women with diochorionic twins. Preeclampsia complicated pregnancy in $26(30.2 \%)$ and 43 (23.6\%) patients, respectively, with monochorionic and diochorionic placentation type. A frequent complication of both the $\mathrm{MH}$ and $\mathrm{DH}$ twins was anemia, which is found in more than half of the surveyed (56.9 and $51 \%$, respectively).

Diagnosis of dissociated fetal growth in multiple pregnancies is based on the difference of bio parietal diameter (BPD) (over $6 \mathrm{~mm}$ ), abdominal circumference (AC) (20 mm), the length of the thigh (LT) $(5 \mathrm{~mm})$ difference of presumed fetal weight (more than $20 \%$ ). During our observations with almost the same frequency of anemia, preeclampsia, and threatened abortion it draws attention the high percentage of dissociated fetal growth during pregnancy MH (22 vs. $14 \%$ ), possibly due to the hemodynamic imbalance. This is consistent with the data of M. Houlton et. al. accordingly; there is a high percentage of ISG at MH double (34\%). With the progression of pregnancy the number of fetuses with growth retardation in $\mathrm{MH}$ type and in $\mathrm{DH}$ placentation increased from $32 \%$ within 32-35 weeks of gestation to $65.9 \%$ within $36-39$ weeks. This FGR of one of the twins was more frequently observed in male fetuses than in females (73.6 vs. $26.4 \%$ ); we didn't find the differences in the frequency of FGR 1st and 2nd fetus (60.8 and $62 \%)$.

Analysis of perinatal outcomes was conducted by taking into account the timing and method of delivery at 182 women with DH (antenatal death of one of the fetus of the two patients) and at 86 patients with $\mathrm{MH}$ with twins (five of SFFT severe miscarriage occurred later in 23-27 weeks of pregnancy).

Urgent deliveries occurred at 29 of 86 (33.7 \%) patients with pregnancy - MH, which majority (24) pregnant women in term 37,5-38,5 weeks of pregnancy. Preterm birth is the most common complication of multiple pregnancies, and occurred in 57 (66.3\%) patients with pregnancy - MH. At DH twins the percentage of preterm births was slightly lower and amounted to 79 (43.4\%). Vaginally resolved $60.5 \%$ of pregnant women with $\mathrm{MH}$ twins and $45 \%$ of pregnant women with $\mathrm{DH}$ placentation type. It should be noted that all women who gave birth vaginally, the first fetus was located only in the cephalic presentation.

The most frequent indications for operative delivery were first breech fetus, fetal distress during pregnancy and childbirth, FGR fetus/fetus, severe preeclampsia, premature detachment of normally situated placenta, anomalies of labor activity. Thus perinatal loss equally observed both after cesarean section and after spontaneous delivery and made $4 \% \mathrm{MH}$ and $1.7 \% \mathrm{DH}$ pregnancies, respectively.

Analysis of the newborn state, depending on the type of placentation shows that monochorionic pregnancy status of children at birth was estimated five times more often below 7 scores at Apgar's scale. This is mainly due to the marked physiological immaturity, malnutrition, asphyxia varying degrees. It should be taken into account the fact that the second MH fetus suffers more.

Manifestations of the immaturity of the lungs were observed at babies born before 37 weeks of gestation. However, according to the same frequency of respiratory distress syndrome (RDS) for the $\mathrm{MH}$ and DH twins, the severity of their manifestations were more pronounced when mono- 
chorionic pregnancy. The number of infants, who required mechanical ventilation after birth, was $15 \%$ in diochorionic twins against $24 \%$ in monochorionic.

The presence of cerebral pathology, according neuron sonography was not directly connected with the method of delivery, however, it is important when it occurred in gestational age when were non-traumatic hemorrhage in newborns, and related complications such as fetal distress, SFFG. Severe cerebral lesion is usually not met, after 33 weeks of gestation in both types of multiple pregnancies. At the same time, we should emphasize the expressed dependence of the frequency of identified lesions on chorionic (among 11 children with severe cerebral lesions were 7 with MH twins, and one - with monochorionic, mono amniotic).

We carried out a study on the development of children with remote $\mathrm{DH}$ and $\mathrm{MH}$ twins at the age of six months. There have been four cases of neonatal mortality in preterm infants with monochorionic twins born 28-34 weeks of gestation and died at the age of 2-3 months. At postmortem examination revealed a complete softening of the brain substance, hemorrhagic hydrocephalus, necrotizing enterocolitis. The presence of cerebral pathology as mild to moderate (perinatal hypoxic lesions of the central nervous system (CNS) disorders, movement disorders syndrome of increased neuron-reflex excitability syndrome, muscular dystrophy), regardless of the delivery method are found in six full-term and preterm infants.

At DH pregnancy complication rate of the CNS in children under six months of life was almost identical and amounted to $27 \%$ against $23 \%$ - with the $\mathrm{MH}$ placentation. Emphasis is made on severe neurological complications in the form of hemi tetraparesis in DH twins, who were born at 32 and 37 weeks of gestation.

Thus, the multiple pregnancies - both $\mathrm{MH}$ and $\mathrm{DH}$ - are a high risk factor for perinatal complications. Careful observation of pregnant women with multiple pregnancy in the antenatal period, the prevention of the most frequent complications of multiple pregnancy (premature labor, anemia, preeclampsia, FGR), ultrasound monitoring with early pregnancy allow for a differentiated approach to the management of pregnancy and childbirth, helping to reduce perinatal morbidity and mortality.

\section{Conclusions}

1. Multiple pregnancies occur with a large number of complications compared with singletons. The threat of abortion was noted in $59.3 \%$ of women with monochorionic and $62 \%$ of women with diochorionic twins, which is three times higher than in singleton pregnancies.

2. Preeclampsia complicated pregnancy in $30.2 \%$ and $23.6 \%$ of patients, $y$, with monochorionic and diochorionic placentation type, respectively, which is two times higher than in singleton pregnancies. A frequent complication of a monochorionic and diochorionic pregnancy anemia was detected in more than half of observations. Noteworthy is a high percentage of fetus growth dissociated with monochorionic pregnancies (22\% vs. $14 \%$ with diochorionic twins).

3. Delivery in the case of multiple pregnancies is also much more complicated and is terminated surgically three times more often than in singleton births. The most frequent indications for operative delivery were first breech fetus, fetal distress during pregnancy and childbirth, fetal growth retardation, severe preeclampsia, premature detachment of normally situated placenta, anomalies of labor activity. These perinatal losses are observed equally both after cesarean section and after spontaneous labor and amounted to $4 \%$ in monochorionic and $1.7 \%$ in diochorionic twins.

4. The prospects for further research are to study the deepening problems antenatal care in multiple pregnancies, the introduction of a mandatory ultrasound in the first trimester of pregnancy with the definition of the type of placentation and the inclusion of a mandatory transvaginal cervimeter surveys algorithm in multiple pregnancies. Thus, the increase in the frequency of multiple pregnancy influences the obstetric and perinatal indicators, pushing this issue in a number of the most pressing issues of modern obstetrics and perinatology.

\section{References}

[1] Ailamazyan, E. K., Kulakov, V. I., Radzinsky, V. E., Savelyev, G. M. (2009). Obstetrics: national leadership. Moscow: GEOTAR Media, 1200. 
[2] Almonte, L., Davis, M., Ward, C., Brown, D., Craparo, F. (2012). Spontaneous and non - spontaneous twins: a comparasion study of preterm labor, preterm premature rupture of membranes, gestational age at delivery, maternal age, and len th of hospital stay. Twin Research and Human Genetics, 15 (2), 170.

[3] Rebrova, O. Y. (2002). Statistical analysis of medical data. The use of the application package STATISTICA. Moscow: Media Sphere, 312.

[4] Hasegawa, I., Tanaka, K., Takahashi, K., Tanaka, T., Aoki, K., Torii, Y. et. al. (1996). Transvaginal ultrasonographic cervical assessment for the prediction of preterm delivery. Journal of Maternal-Fetal and Neonatal Medicine, 5 (6), 305-309. doi: 10.3109/14767059609018404

[5] Heath, V. C. F., Southall, T. R., Souka, A. P., Elisseou, A., Nicolaides, K. H. (1998). Cervical length at 23 weeks of gestation: prediction of spontaneous preterm delivery. Ultrasound in Obstetrics and Gynecology, 12 (5), 312-317. doi: 10.1046/j.1469-0705.1998.12050312.x

[6] Komáromy, B., Lampé, L. (1977). The Value of Bed Rest in Twin Pregnancies. International Journal of Gynecology \& Obstetrics, 15 (3), 262-266. doi: 10.1002/j.1879-3479.1977.tb00689.x

[7] Hypertension in Pregnancy (2013). Obstetrics \& Gynecology, 122 (5), 1122-1131. doi: 10.1097/01. aog.0000437382.03963.88

[8] Podobnik, M., Bulié, M., Smiljanicé, N., Bistrički, J. (1988). Ultrasonography in the detection of cervical incompetency. Journal of Clinical Ultrasound, 16 (6), 383-391. doi: 10.1002/jcu.1870160604

[9] Romero, R., Nicolaides, K. H., Conde-Agudelo, A., O’Brien, J. M., Cetingoz, E., Da Fonseca, E. et. al. (2016). Vaginal progesterone decreases preterm birth $\leq 34$ weeks of gestation in women with a singleton pregnancy and a short cervix: an updated meta-analysis including data from the OPPTIMUM study. Ultrasound in Obstetrics \& Gynecology, 48 (3), 308-317. doi: 10.1002/uog.15953

[10] Wahabi, H. A., Abed Althagafi, N. F., Elawad, M., Al Zeidan, R. A. (2011). Progestogen for treating threatened miscarriage. Cochrane Database of Systematic Reviews. doi: 10.1002/14651858.cd005943.pub3

[11] Smith, C. V., Anderson, J. C., Matamoros, A., Rayburn, W. F. (1992). Transvaginal sonography of cervical width and length during pregnancy. Journal of Ultrasound in Medicine, 11 (9), 465-467. doi: 10.7863/jum.1992.11.9.465

[12] Soneck, J. D., Lams, J. D., Blumenfeld, M. et. al. (1990). Measurement of cervical length in pregnancy: comparison between vaginal ultrasonography and digital examination. Obstetrics \& Gynecology, 76 (2), 172-175.

[13] Cunningham, F. G., Leveno, K. J., Bloom, S. L., Hauth, J. C., Rouse, D. J., Catherine, Y. S. (2010). Hypertensive disorders in pregnancy. Williams Obstetrics. New York: Mc Graw Hill Companies, 1376.

[14] Spellacy, W. (1988). Multiple pregnancies. Danforth's Obstetrics and Gynecology, 891-903.

[15] Timor-Trisch, I. E., Monteagudo, A. (2008). Sonographic evaluation of the cervix in pregnancy. Textbook of Perinatal Medicine. The Parthenon Publishing Group, 374-385.

[16] Wennerholm, U.-B., Holm, B., Mattsby-Baltzer, I., Nielsen, T., Platz-Christensen, J., Sundell, G. et. al. (1997). Fetal fibronectin, endotoxin, bacterial vaginosis and cervical length as predictors of preterm birth and neonatal morbidity in twin pregnancies. BJOG: An International Journal of Obstetrics and Gynaecology, 104 (12), 1398-1404. doi: 10.1111/j.1471-0528.1997.tb11010.x

[17] Wong, G., Levine, D., Ludmir, J. (1997). Maternal postural challenge as a functional test for cervical incompetence. Journal of Ultrasound in Medicine, 16 (3), 169-175. doi: 10.7863/jum.1997.16.3.169

[18] Botting, B. J., Davies, I. M., Macfarlane, A. J. (1987). Recent trends in the incidence of multiple births and associated mortality. Archives of Disease in Childhood, 62 (9), 941-950. doi: 10.1136/adc.62.9.941

[19] Bowie, J., Andreotti, R., Rosenberg, E. (1983). Sonographic appearance of the uterine cervix in pregnancy: the vertical cervix. American Journal of Roentgenology, 140 (4), 737-740. doi: 10.2214/ ajr.140.4.737

[20] Brook, I., Feingold, M., Schwartz, A., Zakut, H. (1981). Ultrasonography in the diagnosis of cervical incompetence in pregnancy-a new diagnostic approach. BJOG: An International Journal of Obstetrics and Gynaecology, 88 (6), 640-643. doi: 10.1111/j.1471-0528.1981.tb01222.x 